\title{
Nonselective Three-Dimensional NMR Spectroscopy. The 3D NOE-HOHAHA Experiment
}

\author{
G. W. Vuister, R. BOELENS, AND R. KAPTEIN \\ Department of Chemistry, University of Utrecht, Padualaan 8, 3584 CH Utrecht. The Netherlands
}

Received May 20, 1988

The addition of a third frequency domain is a natural extension of two-dimensional NMR techniques. Just as 2D NMR spectroscopy has provided a breakthrough in the structure analysis of small biomolecules, it can be expected that three-dimensional NMR spectroscopy will again extend the range of molecules amenable to NMR study. In the 2D techniques, such as COSY, TOCSY, or 2D HOHAHA and NOESY, correlation of two frequency domains is obtained through some interaction such as $J$ coupling, cross relaxation, or chemical exchange $(1)$. In the case of small proteins and oligonucleotides, 2D NMR made it possible to completely assign the high-resolution proton NMR spectra and to use this information for structure analysis (2). However, for larger biomolecules the overlap of resonances presents a serious problem. The introduction of a third frequency domain could then provide the required increase in resolution.

Early cxamples of 3D NMR include 3D zeugmatography (3), a hetcronuclcar ${ }^{13} \mathrm{C}-{ }^{1} \mathrm{H}$ correlation experiment $(4)$, and the "accordion" experiment $(5,6)$. Threedimensional NMR spectroscopy can be regarded as a combination of two $2 \mathrm{D}$ experiments (7). Several of these combinations have recently been reported, such as the homonuclear 3D $J$-resolved experiment ( 8 ), 3D soft COSY-COSY (7), soft NOESYCOSY (9), and soft NOESY-HOHAHA (10). In all these cases data matrices and measuring time were restricted either by chemical-shift elimination in one time domain $(9)$ or by the use of selective pulses $(7,10,11)$. Here we report a $3 \mathrm{D}$ NOEHOHAHA experiment which does not have this limitation. It contains the full chemical-shift information in all three frequency domains. In contrast to a general opinion $(10,11)$ such an experiment can be done within a reasonable time. We apply the method on a relatively large protein, pike parvalbumin, which contains 108 amino acid residues.

The 3D NOE-HOHAHA experiment can be visualized as a combination of a NOESY (12) and a TOCSY or 2D HOHAHA experiment (13-15), as shown in Fig. 1. The FID in the time domain $t_{3}$ is recorded as a function of two variable times $t_{1}$ and $t_{2}$ which are independently incremented. Fourier transformation in three dimensions thus yields spectra which are a function of three independent frequencies. The pulse after the NOE mixing period $\tau_{m}$ must be phase cycled in order to suppress all coherences $p \neq 0$. A phase cycle of four steps on this pulse will achieve this selection for coherences up to $p=3(16)$, which is sufficient in practice. Isotropic mixing induced 


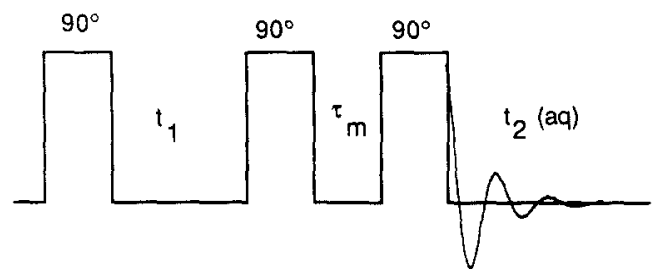

a
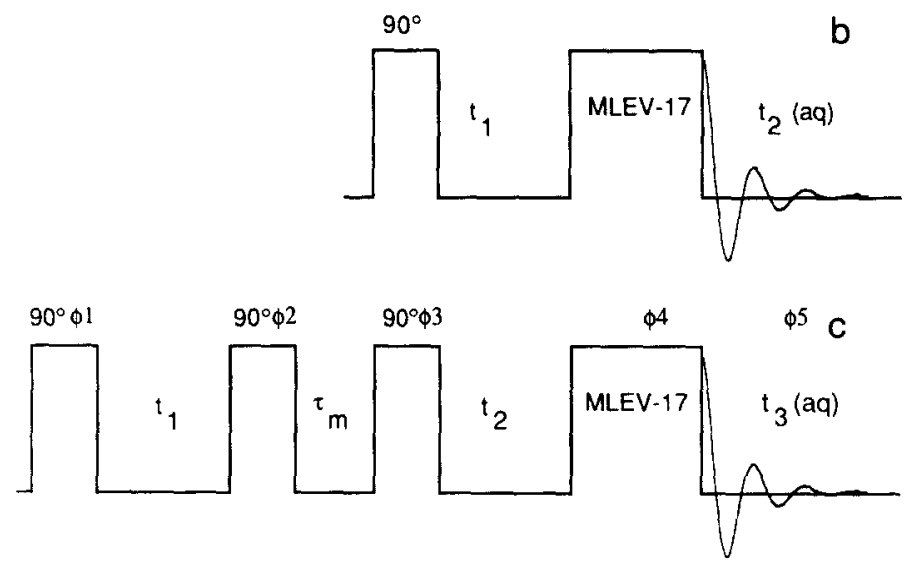

FIG. 1. Pulse sequence for a 3D NOE-HOHAHA experiment (c), constructed from a NOESY experiment (a) and a 2D HOHAHA experiment (b). The corresponding phase cycle is listed in Table 1.

by the MLEV-17 does not involve any change in coherence level. Therefore only axial peaks must be suppressed. The resulting minimum phase cycle is listed in Table 1. Independent time proportional phase increment (TPPI) (16) was used on the $90^{\circ}$ pulses prior to $t_{1}$ and $t_{2}$ in order to separate positive and negative frequencies in these domains.

TABLE 1

Phase Cycle Used in the 3D

NOE-HOHAHA Experiment

\begin{tabular}{ccrrr}
\hline $\begin{array}{c}\phi 1 \\
(+\mathrm{TPPI})\end{array}$ & $\phi 2$ & $\begin{array}{c}\phi 3 \\
\text { (+TPPI) }\end{array}$ & \multicolumn{1}{c}{$\phi$} & $\phi \mathrm{aq}$ \\
\hline$x$ & $x$ & $x$ & $-y$ & $x$ \\
$x$ & $x$ & $y$ & $x$ & $y$ \\
$x$ & $x$ & $-x$ & $y$ & $-x$ \\
$x$ & $x$ & $-y$ & $-x$ & $-y$ \\
$-x$ & $x$ & $x$ & $-y$ & $-x$ \\
$-x$ & $x$ & $y$ & $x$ & $-y$ \\
$-x$ & $x$ & $-x$ & $y$ & $x$ \\
$-x$ & $x$ & $-y$ & $x$ & $y$ \\
\hline
\end{tabular}


The 3D NOE-HOHAHA spectrum was recorded at $500 \mathrm{MHz}$ on a Bruker AM500 spectrometer, equipped with an Aspect 3000 computer. For each FID eight scans were taken, preceded by two dummy scans. The FIDs of 512 words were recorded with the carrier in the middle of the spectrum. During the relaxation delay of $0.6 \mathrm{~s}$ the $\mathrm{H}_{2} \mathrm{O}$ line was suppressed by irradiation. This was repeated for $256 t_{2}$ values and $208 t_{1}$ values, resulting in a total measuring time of $170.5 \mathrm{~h}$. The spectral width in all domains was $6250 \mathrm{~Hz}$. The mixing time $\tau_{\mathrm{m}}$ to allow for NOE magnetization transfer was $150 \mathrm{~ms}$. A MLEV-17 sequence of $47.2 \mathrm{~ms}$ was used for homonuclear HartmanHahn transfer. Two trim pulses at the beginning and end of the MLEV mixing time were used to obtain phase-sensitive spectra $(14,15)$.

The experimental data set of $208 \times 256 \times 512$ points was processed on a microVAX II by software written in Fortran-77. In order to avoid the need of transposition of this large data set from one domain to another, a data structure based on submatrices was used. This allowed fast access to the data in all three dimensions. The data were zero-filled once in the $t_{2}$ and $t_{1}$ time domains prior to the Fourier transformations. Cosine-bell windows were used in all three time domains. Windowing, Fourier transformation, and phasing of all three time domains took a total processing time of $12.5 \mathrm{~h}$. The data were baseline corrected in all frequency domains independently by an automatic baseline correction algorithm using a third-order polynomial fit, which took another $7.5 \mathrm{~h}$ of processing time.

The resulting real spectral data set was $256 \times 256 \times 256$ points. A small fraction of the data points was selected for display on an Evans \& Sutherland PS 300 picture system. This allowed us to obtain a general overview of the 3D spectrum. For interpretation, however, contour plots of cross sections perpendicular to all axes were made.

In the $3 \mathrm{D}$ spectrum three different types of peaks can be identified. Magnetization of spin $a$, which is not transferred during any of the mixing periods, will be found on the body diagonal $\left(\omega_{1}, \omega_{2}, \omega_{3}\right)=\left(\omega_{a}, \omega_{a}, \omega_{a}\right)$. Cross peaks $\left(\omega_{1}, \omega_{2}, \omega_{3}\right)=\left(\omega_{a}, \omega_{b}, \omega_{c}\right)$
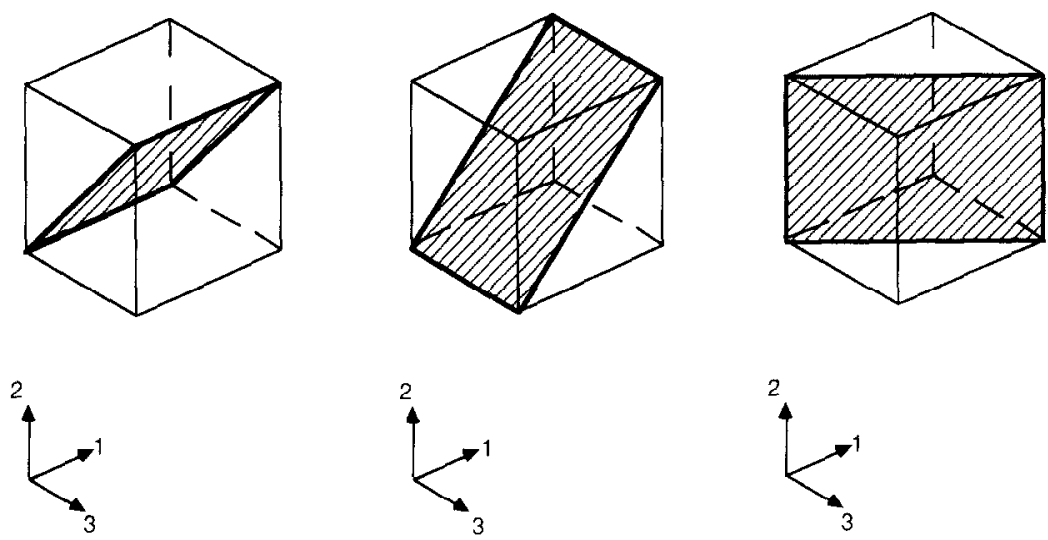

FiG. 2. Schematic representation of the NOE plane (a), the HOHAHA plane (b), and the back transfer plane (c) in a 3D NOE-HOHAHA spectrum. 


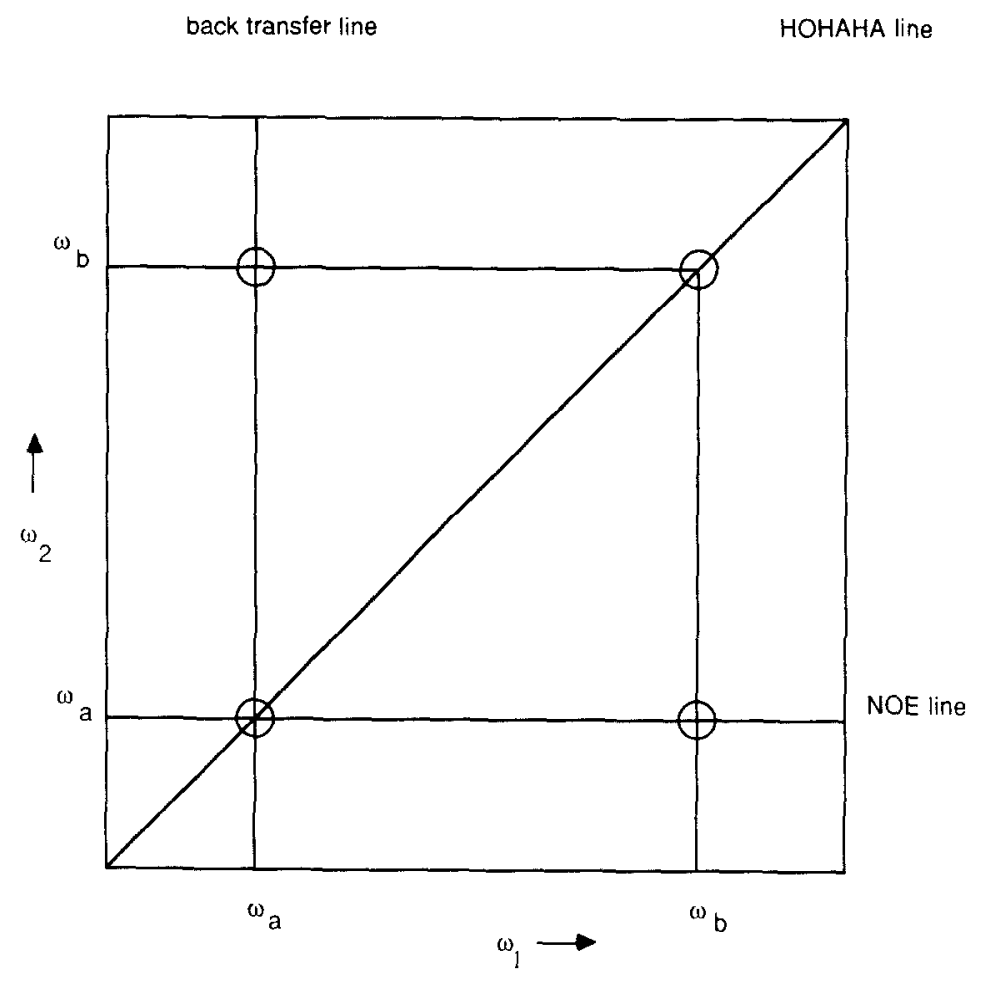

FIG. 3. Schematic representation of a cross section perpendicular to the $\omega_{3}$ axis at $\omega_{3}=\omega_{a}$ in a 3D NOEHOHAHA spectrum. The NOE, HOHAHA, and back transfer lines correspond to the intersections of the corresponding planes with this cross section.

contain the magnetization transferred from spin $a$ to spin $b$ during the NOE mixing period, and from spin $b$ to spin $c$ during the HOHAHA mixing period. The crossdiagonal peaks have two identical frequencies, and a third which is different. There are three types of cross-diagonal peaks, each type located on a different plane in the 3D frequency space (see Fig. 2). The cross-diagonal peaks $\left(\omega_{1}, \omega_{2}, \omega_{3}\right)=\left(\omega_{a}, \omega_{b}, \omega_{b}\right)$ contain magnetization transferred from spin $a$ to spin $b$ only during the NOE mixing period. These peaks are located on the NOE plane $\omega_{2}=\omega_{3}$ (see Fig. 2a). The HOHAHA plane $\omega_{1}=\omega_{2}$ (see Fig. 2b) contains the cross-diagonal peaks $\left(\omega_{1}\right.$, $\left.\omega_{2}, \omega_{3}\right)=\left(\omega_{a}, \omega_{a}, \omega_{b}\right)$, which indicate magnetization transferred from spin $a$ to spin $b$ during the HOHAHA mixing period only. Finally, the cross-diagonal points $\left(\omega_{1}, \omega_{2}, \omega_{3}\right)=\left(\omega_{a}, \omega_{b}, \omega_{a}\right)$ (see Fig. 2c) contain magnetization which is transferred from spin $a$ to spin $b$ during the NOE mixing period, and back from spin $b$ to spin $a$ during the HOHAHA mixing period. These points are located on the so-called back transfer plane $\omega_{1}=\omega_{3}$. The closest 2D analog is the DQ-NOESY experiment ( $l 7$ ), which contains the information of the back transfer plane as a subset. Naturally, all 
three planes contain the diagonal axis $\left(\omega_{1}=\omega_{2}=\omega_{3}\right)$. Any cross section perpendicular to some axis of the $3 \mathrm{D}$ frequency space will intersect with these three planes, resulting in NOE, HOHAHA, and back transfer lines. These three lines cross at the diagonal point, as schematically shown in Fig. 3 for a cross section perpendicular to the $\omega_{3}$ axis.

Figure 4a shows the nonselective 3D NOE-HOHAHA spectrum of parvalbumin in $\mathrm{H}_{2} \mathrm{O}$. It is clear that the spectrum contains a large number of cross peaks (more then 50,000 were found!). Most of the intensity accumulates on the body diagonal (Fig. 4a) and on the three diagonal planes, as can be seen in Fig. 4b, which shows a view of the $3 \mathrm{D}$ cube along its body diagonal. These three planes correspond to the NOE, HOHAHA, and back transfer planes as shown in Fig. 2. The peaks visible between these planes in Fig. $4 \mathrm{~b}$ identify cross peaks at positions $\left(\omega_{3} \neq \omega_{2} \neq \omega_{1}\right)$ and must correspond to magnetization which has been transferred during both mixing periods.

The contour plots of the three planes are shown in Fig. 5. The NOE and HOHAHA planes contain the usual regions with cross peaks, as expected from NOESY and 2D HOHAHA spectra of proteins in $\mathrm{H}_{2} \mathrm{O}$. They also indicate the complexity of the spectra of a protein of 108 amino acids. A unique feature of the present $3 \mathrm{D}$ experiment is the information contained in the back transfer plane. In the upper left quadrant of Fig. $5 \mathrm{c}$ cross-diagonal peaks are visible that represent magnetization transfer from a $\mathrm{NH}$ (i) proton to its own $\mathrm{C}_{\alpha} \mathrm{H}$ (i) proton by NOE, and then back to the same $\mathrm{NH}$ (i) by the isotropic HOHAHA mixing. In the upper right quadrant similar cross-diagonal peaks are found which indicate $\mathrm{C}_{\alpha} \mathrm{H}(\mathrm{i})-(\mathrm{NOE})-\mathrm{C}_{\beta} \mathrm{H}(\mathrm{i})-(\mathrm{HOHAHA})-\mathrm{C}_{\alpha} \mathrm{H}(\mathrm{i})$ transfer, as well as $\mathrm{C}_{\beta} \mathrm{H}(\mathrm{i})-(\mathrm{NOE})-\mathrm{C}_{\alpha} \mathrm{H}(\mathrm{i})-(\mathrm{HOHAHA})-\mathrm{C}_{\beta} \mathrm{H}(\mathrm{i})$ magnetization transfer and other aliphatic connectivities. Back transfer peaks turn out to be a very useful aid in identifying spin systems.

As an example of the usefulness of three-dimensional spectroscopy for the assignment of large molecules, we shall discuss the assignment of a sequence of six amino acids from a 3D NOE-HOHAHA spectrum of parvalbumin in $\mathrm{H}_{2} \mathrm{O}$. Six cross sections perpendicular to the $\omega_{3}$ frequency axis at the $\mathrm{NH}$ frequencies are shown in Fig. 6. For complete elucidation of the spin systems cross sections at the $\mathrm{C}_{\alpha} \mathrm{H}$ and $\mathrm{C}_{\beta} \mathrm{H}$ frequencies were sometimes also used (not shown). Starting at the cross section belonging to Asp 92 (upper left cross section of Fig. 6), a strong peak shows up where the body diagonal intersects with this cross section. This defines the frequency of the NH proton of Asp 92. The diagonal line in this cross section corresponds to the HOHAHA line ( see also Fig. 3) and shows cross-diagonal peaks indicating magnetization transfer from the protons in the Asp 92 spin system to its NH proton. In this way the position of the $\mathrm{C}_{\alpha} \mathrm{H}$ proton can be found. A cross peak on a horizontal line at this frequency defines the presence of a NOE to this $\mathrm{C}_{\alpha} \mathrm{H}$ proton. Thus the back transfer to its own NH, as well as NOEs to the $\beta$ protons, can be observed. An additional NOE in the $\mathrm{NH}$ region corresponds to the sequential $d_{\alpha \mathrm{N}}$ connectivity to the $\mathrm{NH}$ of Gly 93. The sequential step is indicated by a vertical line connecting this cross section with the cross section in the middle left of Fig. 6 . This cross section at the NH frequency of Gly 93 shows a similar analysis leading to the next sequential step to Asp 94. This continues to Gly 95, which again shows the typical pattern for a glycine spin system. For the next sequential step the $d_{\mathrm{NN}}$ NOE to the amide proton of Lys 96 was used 

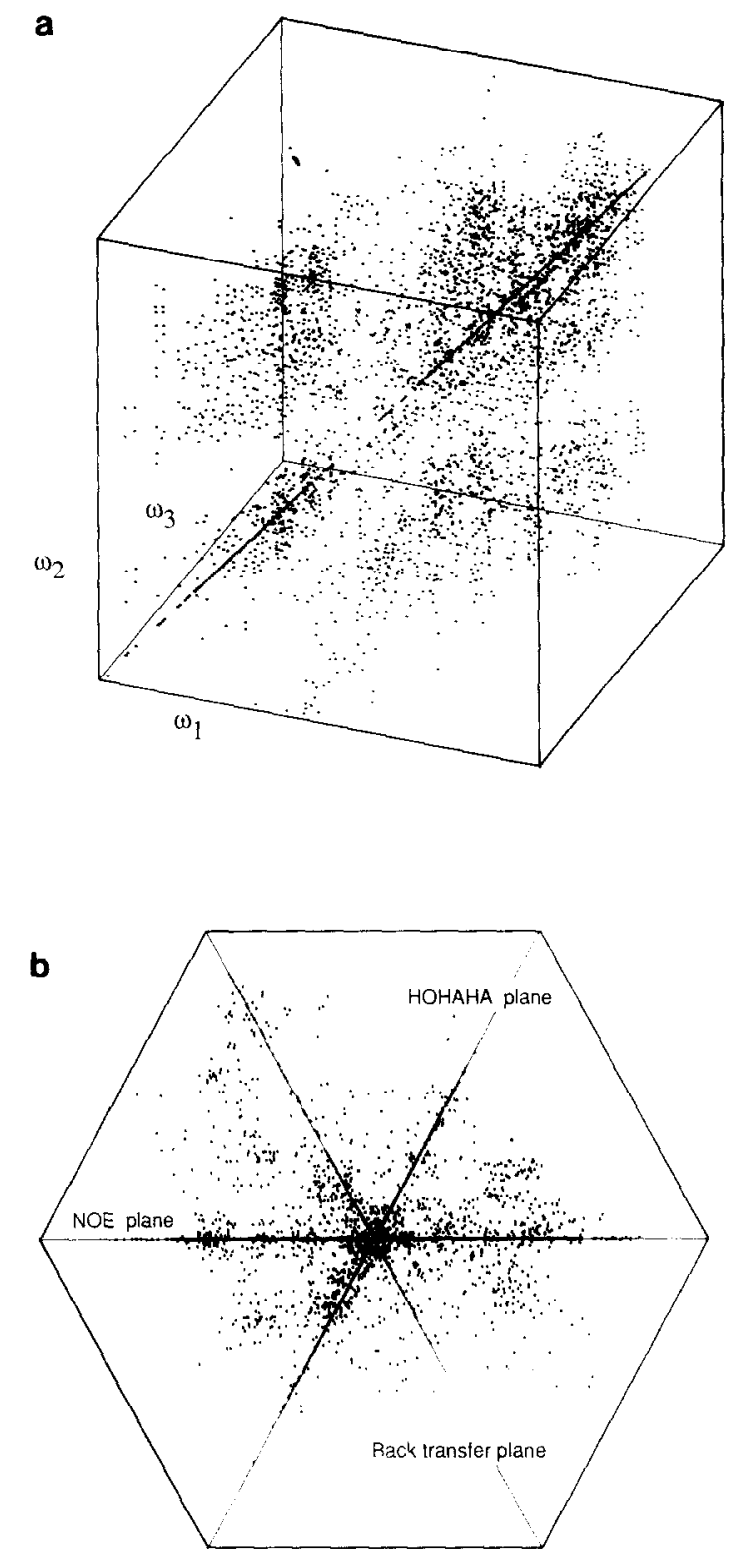

Fig. 4. 3D NOE-HOHAHA spectrum of $8.7 \mathrm{~m} M$ pike parvalbumin $(\mathrm{p} I=5.0)$ prepared as described previously (18). The spectrum was recorded in a $90 / 10(\mathrm{v} / \mathrm{v}) \mathrm{H}_{2} \mathrm{O} / \mathrm{D}_{2} \mathrm{O}$ mixture at $\mathrm{pH} 4.1$ at $315 \mathrm{~K}$. Parts (a) and (b) show local maxima of the spectrum. The local maxima were obtained by searching within a small volume for the position of the maximum intensity above a threshold. In this way more than 50,000 peaks were found. Only a small fraction of these peaks were plotted in (a) and (b). The plane containing the $t_{1}, t_{2}$ noise of the strong $\mathrm{H}_{2} \mathrm{O}$ resonance was eliminated. (a) View of the 3D cube of the 3D NOEHOHAHA spectrum and (b) view along the diagonal axis $\left(\omega_{3}=\omega_{2}=\omega_{1}\right)$ of the $3 \mathrm{D}$ cube. 

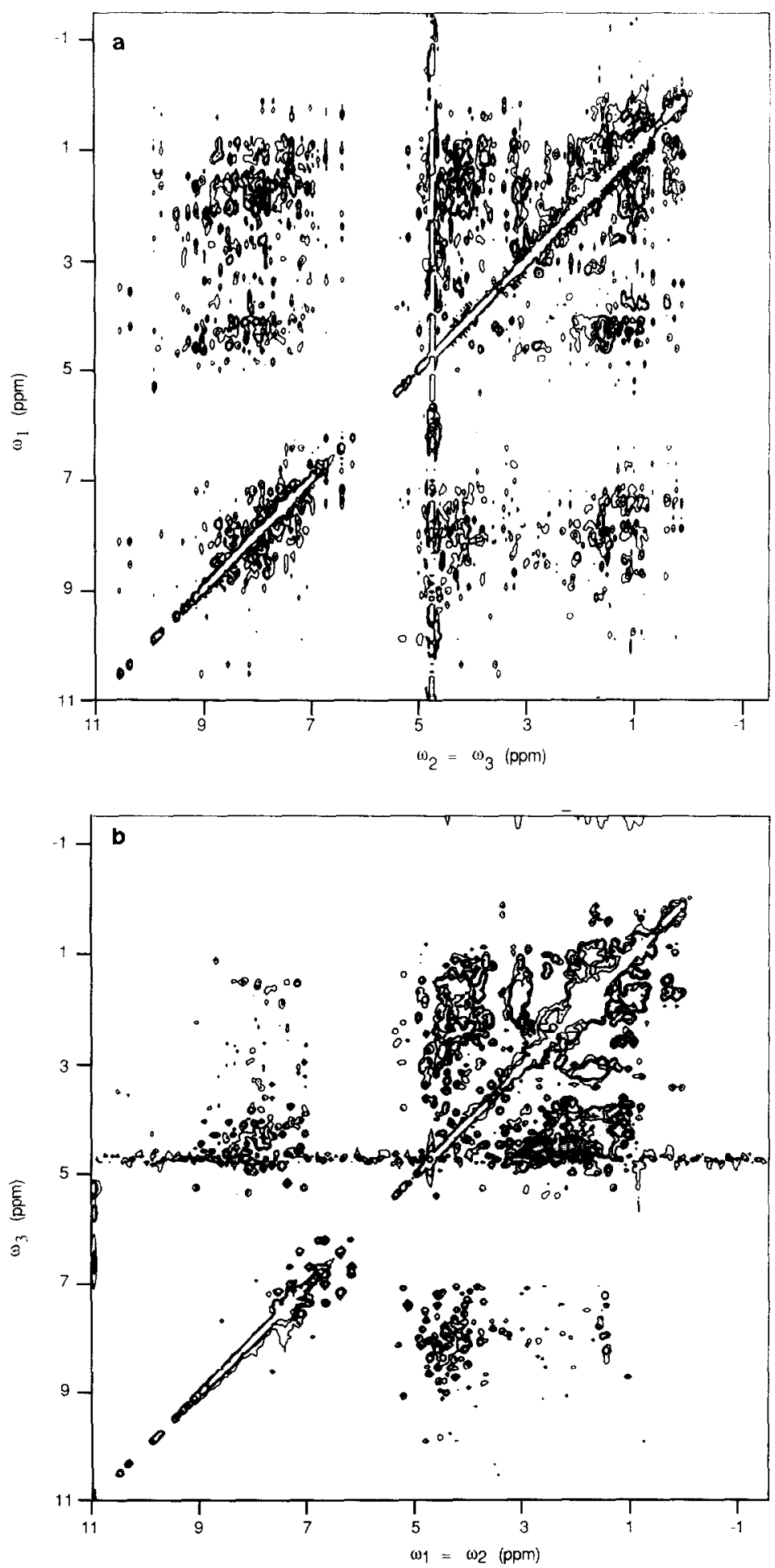

FIG. 5. Diagonal planes of a 3D NOE-HOHAHA spectrum of parvalbumin in $\mathrm{H}_{2} \mathrm{O}$. (a) NOE plane, (b) HOHAHA plane, and (c) back transfer plane. 


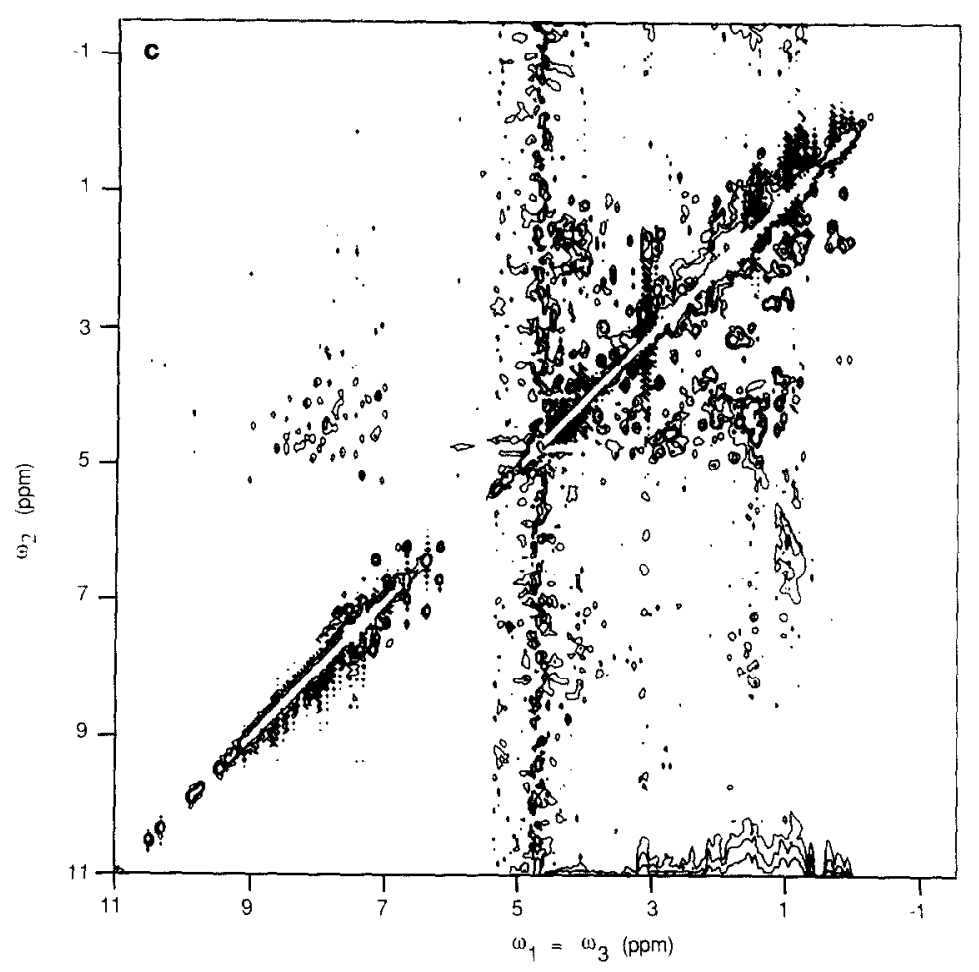

FiG. 5-Continued.

because the $d_{\alpha \mathrm{N}}$ NOE was missing in this case. Finally in the lower right Fig. 6 shows the cross section of the $\mathrm{NH}$ frequency of Ile 97 , which was found via the $d_{\alpha \mathrm{N}}$ connectivity of Lys 96 . The next $d_{\alpha \mathrm{N}}$ sequential connectivity to Gly 98 is also indicated in this cross section. Assignments were in exact agreement with those reported previously (18). A more detailed account concerning assignment strategies in $3 \mathrm{D}$ spectra will be given in a subsequent communication.

The present results show that a 3D NMR spectrum of a protein can be obtained covering the full chemical-shift range of $12.5 \mathrm{ppm}$ in all frequency domains. Of course some digital resolution in the individual time domains is sacrificed compared to $2 \mathrm{D}$ NMR, but this is more than compensated for by the gain in spectral resolution obtained by adding a third frequency domain.

A large fraction (about $25 \%$ ) of the relatively long measuring time was spent on disk I/O operations, loading of the pulse programmer of the Aspect 3000, and dummy scans which could in principle be avoided. Reduction of the measuring time is important because this reduces the chance of instabilities which are expressed as $t_{1}, t_{2}$ noise. Processing time and effort remain within reasonable limits if suitable algorithms are used, which allow easy access to the data in all three dimensions without the need for transposition.

A spectrum in $3 \mathrm{D}$ frequency space contains, in principle, all information necessary for determination and sequential assignment of spin systems. The present experiment 


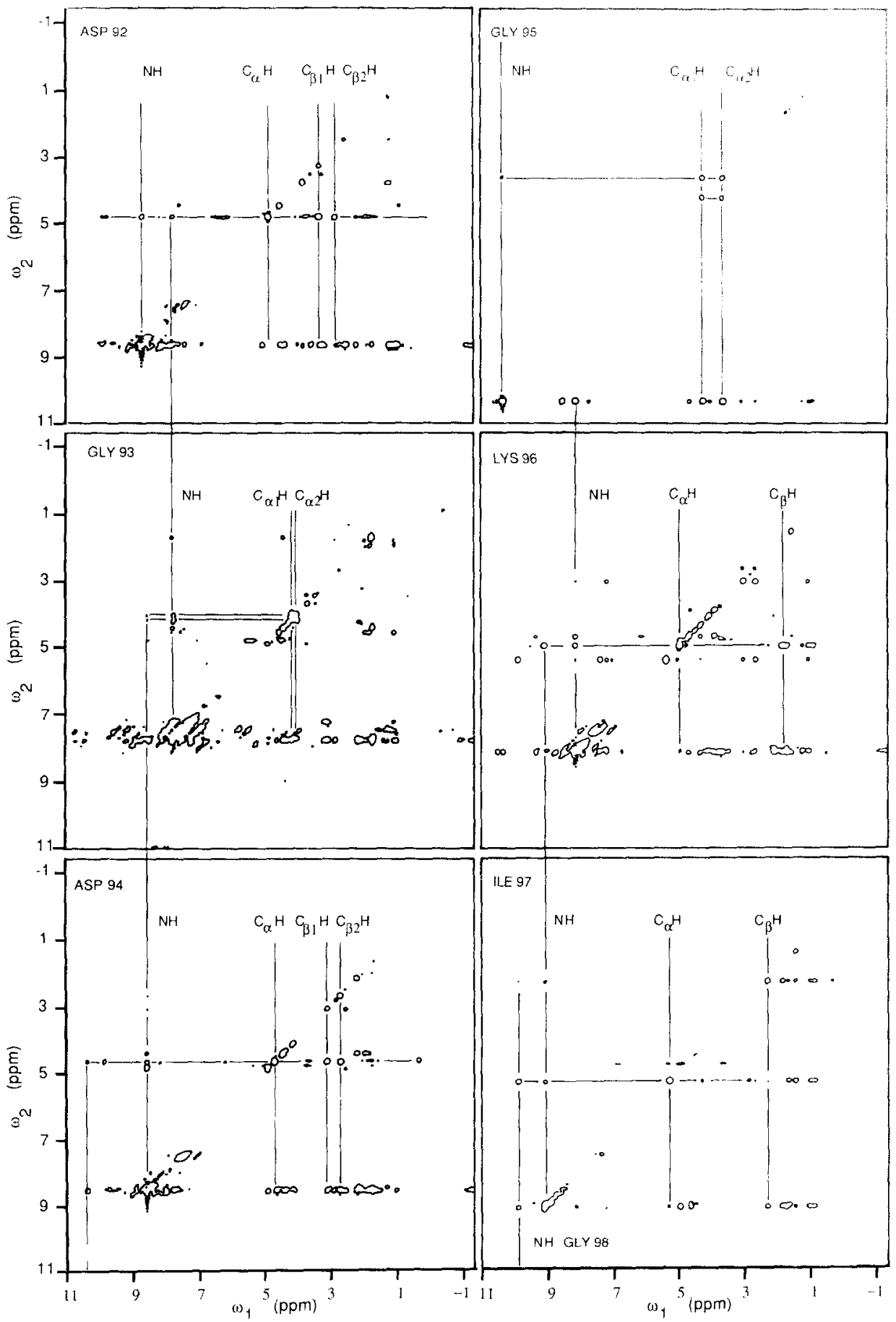

FIG. 6. Cross sections of the 3D NOE-HOHAHA spectrum of pike parvalbumin in $\mathrm{H}_{2} \mathrm{O}$ perpendicular to the $\omega_{3}$ axis at the frequencies of the amide protons of Asp 92 to Ile 97 . Sequential assignment is indicated (see text for explanation). 
contains the NOESY spectrum and the 2D HOHAHA spectrum as subsets. A 3D NOE-HOHAHA experiment simplifies the assignment procedures due to reduction of overlap problems, but also because both mixing phenomena occur under exactly the same conditions. The examples studied indicate the great potential of $3 \mathrm{D}$ spectroscopy for assignment and structure elucidation of large molecules.

\section{ACKNOWLEDGMENTS}

This work was supported by the Netherlands Foundation for Chemical Research (SON) with financial aid of the Netherlands Organization of Scientific Research (NWO). We thank Dr. A. Padilla for his kind gift of the parvalbumin sample and Mr. D. Schreurs for writing the interface software for transfer of the NMR data to the PS 300 picture system.

\section{REFERENCES}

I. R. R. ERnst, G. Bodenhausen, ANd A. Wokaun, "Principles of Nuclear Magnetic Resonance in One and Two Dimensions," Clarendon, Oxford, 1987.

2. K. WÜTHRICH, "NMR of Proteins and Nucleic Acids," Wiley, New York, 1986.

3. A. KUMAR, D. Welti, AND R. R. ERNST, J. Magn. Reson. 18, 69 (1975).

4. P. H. Bolton, J. Magn. Reson. 46, 343 (1982).

5. G. BODENHAUSEN AND R. R. ERNST, J. Magn. Reson. 45, 367 (1981).

6. G. BodenHAUSEN AND R. R. ERNST, J. Am. Chem. Soc. 104, 1304 (1982).

7. C. GRIESINGER, O. W. SøRENSEN, AND R. R. ERNST, J. Magn. Reson. 73, 574 (1987).

8. G. W. Vuister AND R. BoElens, J. Magn. Reson. 73, 328 (1987).

9. C. Griesinger, O. W. SøRensen, AND R. R. ERnSt, J. Am. Chem. Soc. 109, 7227 (1987).

10. H. OsChkinat, C. Griesinger, P. J. Kraulis, O. W. SOREnSEn, R. R. ERnst, A. M. Gronenborn, AND G. M. ClORE, Nature (London) 332, 374 (1988).

11. D. RINGE, Nature (London) 332, 303 (1988).

12. J. Jeener, B. H. Meier, P. Bachmann, AND R. R. Ernst, J. Chem. Phys. 71, 4546 (1979).

13. L. BRAUSCHWEILER AND R. R. ERNST, J. Magn. Reson. 53, 521 (1983).

14. D. G. Davies and A. BAX, J. Am. Chem. Soc. 107, 2820 (1985).

15. A. BAX AND D. G. DAVIES, J. Magn. Reson. 65, 355 (1985).

16. G. BOdENHAuSEN, H. Kogler, AND R. R. ERNST, J. Magn. Reson. 58, 370 (1984).

17. F. J. M. van DeR Ven, C. A. G. HAasnoot, AND C. W. Hilbers, J. Magn. Reson. 61, 181 (1985).

18. A. Padilla, A. Cavé, AND J. Parello, $J$. Mol. Biol., submitted. 